\title{
Multicomponent Cluster States in Dilute Mixed Molecular Crystals, with Application to ${ }^{1} B_{2 u}$ Naphthalene Excitons*
}

\author{
Hwei-Kwan Hong† and RaOul Kopelyan \\ Department of Chemistry, The University of Michigan, Ann Arbor, Michigan 48104
}

(Received 17 May 1972)

\begin{abstract}
We develop the theory of Frenkel excitons for multicomponent cluster states in medium-dilute mixed molecular crystals. This theory can be applied to both secondary host traps induced by a single impurity and to multicompositional, chemical, or isotopic clusters. A Green's function technique, which is a generalization of the Koster-Slater method, is developed and utilized. Symmetry properties of such clusters are discussed, with emphasis on interchange equivalent sites in nonsymmorphic crystals. The optical spectra of naphthalene- $h_{8}$ in naphthalene- $d_{8}$ can now be further analyzed, with the help of some numerical calculations on multicomponent cluster states. Using our recently acquired dispersion relation for the ${ }^{1} B_{2 u}$ naphthalene exciton state we fit satisfactorily both the fine structure and the "hyperfine" structure, without any additional parameters, except for the experimentally known trap-depths of a few isotopic impurities. This corroborates both the importance of the exciton superexchange effect and the validity of the exciton dispersion formula.
\end{abstract}

\section{INTRODUCTION}

In this paper, we investigate the Frenkel exciton ${ }^{1}$ states and optical spectra of multicomponent mixed crystals in which more than one type of guests are present in otherwise "neat" or "ordered" host lattices. The guest molecules discussed here can be either compositionally different from the host, such as impurities in chemical or isotopic mixed crystals, or simply "perturbed" host molecules which are compositionally identical to the rest of the bulk but reside in physically different environments. These guests are assumed to form clusters, with nontrivial interactions among the guests within the cluster. However, the cluster-cluster interactions will be ignored. The concentrations of various guests have to be large enough so that such clusters do exist but not too large to form heavily doped mixed crystals. The present problem, therefore, differs from that of dilute mixed crystals with well separated impurities ${ }^{2}$ and also from that of more concentrated multicomponent systems ${ }^{3}$ which do not have a well-defined major component. Only bound states outside the host band are treated here because they are observed and usually more informative. ${ }^{4}$

The importance of such multicomponent cluster states can be recognized if one realizes that most of the binary mixed crystals such as dilute naphthalene- $h_{8}$ in naphthalene- $d_{8}$ are really multicomponent systems due to the inevitable presence of partially deuterated hosts $\left(\mathrm{C}_{10} \mathrm{D}_{7} \mathrm{H}\right.$ in this case) or even ${ }^{13} \mathrm{C}$ isotopes because of natural abundance (such as ${ }^{13} \mathrm{C}^{12} \mathrm{C}_{9} \mathrm{H}_{8}$ ). In fact, this research was partly prompted by Hanson's ${ }^{5 \mathrm{a}}$ observation that the spectra of dilute naphthalene- $h_{8}$ in $-d_{8}$ mixed crystals contain several doublets. One plausible explanation would be to ascribe such doubling to the presence of multicomponent clusters. This effect is particularly important in the shallow trap case.

Another even more widely occurring case would be the multicomponent systems consisting of guest, perturbed host and host. Admittedly, such systems are difficult to distinguish from the simple guest-host systems if the guest perturbations on the host are small. In some cases, however, such perturbations might play a predominant role. It has been reported by Zalewski and $\mathrm{McClure} \mathrm{fa}^{\mathrm{a}}$ that the multiple structure in the guest phosphorescence spectrum of pyrazine- $h_{4}$ in $-d_{4}$ is due to the anomalous site effect of hydrogen bonding. The same effect may also cause the red shift of a $S-T$ transition of a host that is directly hydrogen-bonded to a guest. In fact, Hong ${ }^{6 b}$ has observed a shallow trap transition ( $7 \mathrm{~cm}^{-1}$ to the red of the host absorption) in a $S \rightarrow T$ absorption spectrum of pyrazine- $h_{4}$ in $-d_{4}$, which can only be assigned as the perturbed host level. Thus, we have multicomponent clusters ( $\mathrm{r}^{7}$ "conglomerates") of guests and perturbed hosts even at the infinitely dilute limit. Again, the presence of such multicomponent clusters is expected to have an important bearing on the energy states of shallow traps such as pyrazine- $d_{3}$ (in pyrazine- $d_{4}$ ).

We shall first develop the theory of energy levels and optical properties of multicomponent impurity cluster states within the general Frenkel formulation. Symmetry properties of such clusters will also be discussed with emphasis on the clusters with interchange equivalent sites. ${ }^{8}$ Finally, optical spectra of isotopicly mixed crystals of naphthalene- $h_{8}$ in naphthalene- $d_{8}$ are further analyzed in the light of present theoretical results. In particular, numerical calculations on the multicomponent cluster states are performed, using the recently acquired dispersion relation ${ }^{56}$ and compared with experimental results. This is done not only to further assign fine structure in the spectra but also to demonstrate the important role of the superexchange effect discussed earlier ${ }^{5 \mathrm{c}}$ in elucidating the exciton interactions, which are responsible for the band structures of both pure and mixed molecular crystals.

\section{GENERAL MIXED CRYSTAL PROBLEM IN LOCALIZED REPRESENTATION}

We shall assume here that molecules comprising the crystal are fixed in the lattice and, therefore, the effect 
of exciton-phonon coupling can be ignored. We shall also deal with "isolated" exciton bands and ignore high order corrections due to band-band interactions. In the tight-bonding approximation, ${ }^{1}$ the wavefunctions for such a nonvibrating molecular crystal can be constructed from the wavefunctions of molecules. In particular, for molecular crystals with more than one molecule per unit cell, a set of wavefunctions representing the localized excitation in the crystal can be written as

$$
\psi^{f}\left(\mathbf{R}_{\mathbf{n} \alpha}\right)=\phi^{f}\left(\mathbf{R}_{\mathbf{n} \alpha}\right) \prod_{\mathrm{m} \beta \neq \mathrm{n} \alpha} \phi^{0}\left(\mathbf{R}_{\mathrm{m} \beta}\right),
$$

where a general site $R_{n \alpha}$ is denoted by a unit cell index $n$ and a sublattice index $\alpha . \phi^{0}$ and $\phi^{f}$ are, respectively, the wavefunctions for the ground state and the $f$ thelectronic excited state of free molecules. ${ }^{9}$ On the other hand, the ground state wavefunction for the crystal can be written as

$$
\psi^{0}=\prod_{\mathbf{n} \alpha} \phi^{0}\left(\mathbf{R}_{\alpha \mathbf{n}}\right)
$$

For the pure crystal, the total Hamiltonian is

$$
H=\sum_{\mathrm{n} \alpha} H_{\mathrm{n} \alpha}+\frac{1}{2} \sum_{\mathrm{n} \alpha \neq \mathrm{m} \beta} V_{\mathrm{n} \alpha, m \beta}
$$

where $H_{n \alpha}$ is the free molecular Hamiltonian and $V_{\mathrm{n} \alpha, \mathrm{m} \beta}$ is the interaction term between molecules at $n \alpha$ and $m \beta$. In order to illustrate how the perturbation enters into our mixed crystal Hamiltonian, we write down explicitly the pure crystal Hamiltonian in the localized representation, using wavefunctions of Eqs. (1) and (2).

Thus, we have

$$
\begin{aligned}
H_{\mathbf{n} \alpha, \mathrm{n} \alpha} f-E^{0} & =\left\langle\psi^{f}\left(\mathbf{R}_{\mathbf{n} \alpha}\right)|H| \psi^{f}\left(\mathbf{R}_{\mathbf{n} \alpha}\right)\right\rangle-\left\langle\psi^{0}|H| \psi^{0}\right\rangle \\
& =\epsilon_{h}^{f}+D_{h}^{f}, \\
H_{\mathrm{n} \alpha, \mathrm{m} \beta^{f}} & =\left\langle\psi^{f}\left(\mathbf{R}_{\mathbf{n} \alpha}\right)|H| \psi^{f}\left(\mathbf{R}_{\mathrm{m} \beta}\right)\right\rangle=v_{\mathrm{n} \alpha, \mathrm{m} \beta},
\end{aligned}
$$

where

$$
\begin{array}{r}
D_{h}^{f}=\sum_{\mathrm{m} \beta}\left[\left\langle\phi_{\mathrm{n} \alpha}{ }^{f} \phi_{\mathrm{m} \beta}{ }^{0}\left|V_{\mathrm{n} \alpha, \mathrm{m} \beta}\right| \phi_{\mathrm{n} \alpha}{ }^{f} \phi_{\mathrm{m} \beta}{ }^{0}\right\rangle\right. \\
\left.-\left\langle\phi_{\mathrm{n} \alpha}{ }^{0} \phi_{\mathrm{m} \beta}\left|V_{\mathrm{n} \alpha, \mathrm{m} \beta}\right| \phi_{\mathrm{n} \alpha}{ }^{0} \phi_{\mathrm{m} \beta}{ }^{0}\right\rangle\right] \\
v_{\mathrm{n} \alpha, \mathrm{m} \beta}=\left\langle\phi_{\mathrm{n} \alpha}{ }^{f} \phi_{\mathrm{m} \beta}{ }^{0}\left|V_{\mathrm{n} \alpha, \mathrm{m} \beta}\right| \phi_{\mathrm{n} \alpha}{ }^{0} \phi_{\mathrm{m} \beta^{f}}\right\rangle
\end{array}
$$

and $\epsilon_{h}{ }^{\prime}$ is the gas-phase transition energy from the ground state to the $f$ th excited state and $D_{h}{ }^{f}$ is the socalled "gas-to-crystal shift" or "site shift", due to the fact that the van der Waals interactions with the environment are different for an excited molecule and an unexcited molecule. $v_{\mathrm{n} \alpha, \mathrm{m} \beta}$ is the dynamic interaction which is responsible for the excitation migration.

The diagonalization of the pure crystal Hamiltonian can be achieved by utilizing the translational invariance of the lattice. For molecular crystals which generally have more than one molecule per unit cell, further construction of linear combinations of one-site Bloch functions is called for. In general,

$$
\psi_{\mu}{ }^{f}(\mathbf{k})=N^{-1 / 2} \sum_{\mathrm{n} \alpha} B_{\mu \alpha}{ }^{f}(\mathbf{k}) \exp \left(i \mathbf{k} \cdot \mathbf{R}_{\mathbf{n} \alpha}\right) \psi^{f}\left(\mathbf{R}_{\mathbf{n} \alpha}\right),
$$

where $N$ is the total number of unit cells in the crystal and the $B_{\mu \alpha}{ }^{f}(\mathbf{k})$ 's are the coefficients, which, in special cases, can be uniquely determined from the unit cell symmetry. Furthermore, for centrosymmetric molecular crystals such as crystalline benzene and naphthalene, the coefficients $B_{\mu \alpha}{ }^{f}(\mathbf{k})$ are always real, if the origin is placed at the inversion center. ${ }^{10}$

Let us consider a mixed crystal, which can be either isotopically mixed or chemically mixed, with a few impurities (guests) imbedded in the host lattice. In order to make the formulation more general we do not restrict ourselves to the simple two-component systems. The guests in question, therefore, do not necessarily belong to one chemical species. Even when guests are compositionally identical they might be physically different inside the crystal because of the different environments they are situated in. Further discussion of this aspect, from a group theoretical point of view, follows in the next section. It is convenient to express the mixed crystal Hamiltonian in terms of the pure crystal Hamiltonian plus perturbations. We have

$$
\begin{aligned}
\mathfrak{F}= & =\sum_{\mathrm{n} \alpha \neq p} H_{\mathrm{n} \alpha}+\sum_{p} H_{p}^{\prime}+\frac{1}{2} \sum_{\mathrm{n} \alpha \neq p, \mathrm{~m} \beta \neq p, \mathrm{n} \alpha \neq \mathrm{m} \beta} V_{\mathrm{n} \alpha, \mathrm{m} \beta} \\
& +\sum_{\mathrm{n} \alpha} \sum_{p} V_{\mathrm{n} \alpha, p}^{\prime}+\frac{1}{2} \sum_{p \neq p^{\prime}} V_{p, p^{\prime}}^{\prime \prime} \\
=H+\Delta, &
\end{aligned}
$$

where

$$
\begin{aligned}
\Delta=\sum_{p}\left(H_{p}{ }^{\prime}-H_{p}\right)+ & \sum_{\mathrm{n} \alpha} \sum_{p}\left(V_{n \alpha, p}^{\prime}-V_{\mathrm{n} \alpha, p}\right) \\
& +\frac{1}{2} \sum_{p \neq p^{\prime}}\left(V_{p, p^{\prime}}^{\prime \prime}-V_{p, p^{\prime}}\right) .
\end{aligned}
$$

We have used $p$ to denote sites occupied by guests. Furthermore, the perturbations are assumed to be delocalized so that both the molecular Hamiltonian and the intersite interactions are changed upon the introduction of guests. $V, V^{\prime}, V^{\prime \prime}$ are respectively, host-host, host-guest, and guest-guest interactions. In isotopic mixed crystals, it is generally assumed that $V=V^{\prime}=V^{\prime \prime}$. However, this is not the case when chemical mixed crystals are involved.

As was pointed out by Craig and Philpott, ${ }^{11}$ two different approaches can be used in evaluating the mixed crystal Hamiltonian matrix with a localized basis set. In the first approach, one could use the host free molecule wavefunctions everywhere, including the guest sites, and thus concentrate only on the perturbation in the Hamiltonian operator. Or, one can replace the host molecular functions with guest wavefunctions whenever there is a guest and thus consider both changes, the change in the Hamiltonian and that in the wavefunctions. These two views are equivalent as long as we stay within the Frenkel formulation, which is basically a first order theory. We shall, in the following, adopt the second approach, because it provides a more coherent picture where general impurities are considered. 


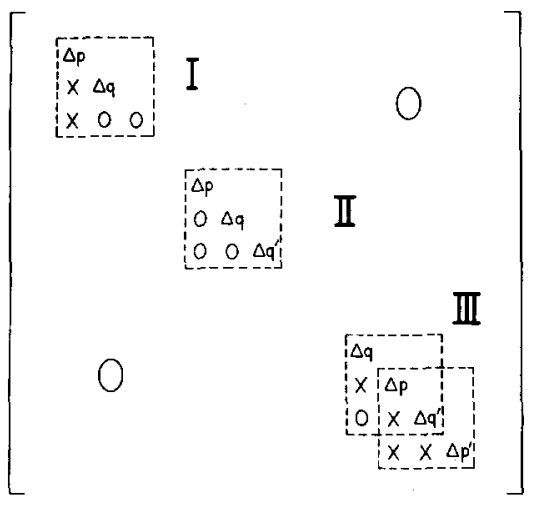

Frg. 1. The general form of the perturbation matrix due to impurities. In I the off-diagonal perturbation extends beyond the diagonal perturbation; in II the opposite happened, and in III the spheres of perturbation due to two impurities overlap.

The perturbation is found to be

$$
\begin{aligned}
& \Delta_{p, p^{f}}=\left\langle\psi^{f}\left(\mathbf{R}_{p}\right)|\Delta| \psi^{f}\left(\mathbf{R}_{p}\right)\right\rangle-\left\langle\psi^{0}|\Delta| \psi^{0}\right\rangle \\
& =\epsilon_{p}^{f}-\epsilon_{h}^{f}+\sum_{q \neq p, q \neq p^{\prime}}\left(\left\langle\bar{\phi}_{p}{ }^{f} \phi_{q}^{0}\left|V_{q, p}^{\prime}\right| \bar{\phi}_{p}{ }^{f} \phi_{q}{ }^{0}\right\rangle\right. \\
& -\left\langle\boldsymbol{\phi}_{p}{ }^{f}{\phi_{q}}{ }^{0}\left|V_{q, p}\right| \phi_{p}{ }^{f} \phi_{q}{ }^{0}\right\rangle-\left\langle\bar{\phi}_{p}{ }^{0} \phi_{q}{ }^{0}\left|V^{\prime}{ }_{q, p}\right| \bar{\phi}_{p}{ }^{0} \phi_{q}{ }^{0}\right\rangle \\
& \left.+\left\langle\phi_{p}{ }^{0} \phi_{q}{ }^{0}\left|V_{q, p}\right| \phi_{p}{ }^{0} \phi_{q}{ }^{0}\right\rangle\right) \\
& +\sum_{p^{\prime} \neq p}\left(\left\langle\bar{\phi}_{p}{ }^{j} \bar{\phi}_{p^{\prime}}\left|V_{p^{\prime}, p}^{\prime \prime}\right| \bar{\phi}_{p}{ }^{f} \bar{\phi}_{p^{\prime}}{ }^{0}\right\rangle\right. \\
& -\left\langle\phi_{p}{ }^{f} \phi_{p^{\prime}}{ }^{0}\left|V_{p^{\prime}, p}\right| \phi_{p}{ }^{f}{\phi_{p^{\prime}}}^{0}\right\rangle-\left\langle\bar{\phi}_{p}{ }^{0} \bar{\phi}_{p^{\prime}}{ }^{0}\left|V^{\prime \prime}{ }_{p^{\prime}, p}\right| \bar{\phi}_{p}{ }^{0} \bar{\phi}_{p^{\prime}}{ }^{0}\right\rangle \\
& \left.+\left\langle\boldsymbol{\phi}_{p}^{0} \boldsymbol{\phi}_{p^{\prime}}{ }^{0}\left|V_{p^{\prime}, p}\right| \boldsymbol{\phi}_{p}{ }^{0} \boldsymbol{\phi}_{p^{\prime}}{ }^{0}\right\rangle\right) \\
& =\Delta_{p}^{f},
\end{aligned}
$$

where $q$ denotes secondary traps (see below). Notice that we have used $\bar{\phi}$ to denote the guest wavefunctions. It should also be pointed out that if another type of guest is present, (say at $p^{\prime}$ ), Eq. (7a) is still valid. Under this condition one would have to understand that $\bar{\phi}_{p^{\prime}}$ in $\mathrm{Eq}$. (7a) is the free molecule wavefunction of the second type of guests.

Similarly,

$$
\begin{aligned}
\Delta_{q, q} f & =\sum_{p}\left(\left\langle\phi_{q}{ }^{f} \bar{\phi}_{p}{ }^{0}\left|V_{q, p}^{\prime}\right| \phi_{q}^{f} \bar{\phi}_{p}{ }^{0}\right\rangle\right. \\
- & \left.\left\langle\phi_{q}{ }^{0} \phi_{p}{ }^{0}\left|V_{q, p}\right| \phi_{q}^{f} \phi_{p}{ }^{0}\right\rangle-\left\langle\phi_{q}{ }^{0} \bar{\phi}_{p}{ }^{0}\left|V_{q, p}^{\prime}\right| \phi_{q}{ }^{0} \bar{\phi}_{p}{ }^{0}\right\rangle\right) \\
& \left.+\left\langle\phi_{q}{ }^{0} \boldsymbol{\phi}_{p}{ }^{0}\left|V_{q, p}\right| \phi_{q}{ }^{0} \phi_{p}{ }^{0}\right\rangle\right)
\end{aligned}
$$

and (see below)

$$
\begin{gathered}
\Delta_{n \alpha, n \alpha}{ }^{f}=\sum_{p}\left(\left\langle\phi_{n \alpha}{ }^{f} \bar{\phi}_{p}{ }^{0}\left|V_{n \alpha, p}^{\prime}\right| \phi_{n \alpha}{ }^{f} \bar{\phi}_{p}{ }^{0}\right\rangle\right. \\
-\left\langle\phi_{n \alpha}{ }^{f} \phi_{p}{ }^{0}\left|V_{n \alpha, p}\right| \phi_{n \alpha}{ }^{f} \phi_{p}{ }^{0}\right\rangle-\left\langle\phi_{n \alpha}{ }^{0} \bar{\phi}_{p}{ }^{0}\left|V_{n \alpha, p}^{\prime}\right| \phi_{n \alpha}{ }^{0} \bar{\phi}_{p}{ }^{0}\right\rangle \\
+ \\
\left.+\left\langle\phi_{n \alpha}{ }^{0} \phi_{p}{ }^{0}\left|V_{n \alpha, p}\right| \phi_{n \alpha}{ }^{0} \phi_{p}{ }^{0}\right\rangle\right) \\
=\Delta_{n a}{ }^{f} \approx 0 .
\end{gathered}
$$

The off-diagonal elements are

$$
\begin{aligned}
& \Delta_{p, p^{\prime}}=\left\langle\bar{\phi}_{p}{ }^{f} \bar{\phi}_{p^{\prime}}{ }^{0}\left|V^{\prime \prime}{ }_{p, p^{\prime}}\right| \bar{\phi}_{p}{ }^{0} \bar{\phi}_{p^{\prime}}{ }^{\prime}\right\rangle \\
& -\left\langle\phi_{p}{ }^{f} \phi_{p^{\prime}}{ }^{0}\left|V_{p, p^{\prime}}\right| \phi_{p}{ }^{0} \phi_{p^{\prime}}\right\rangle, \\
& \Delta_{p, q}{ }^{f}=\left\langle\Phi_{p}{ }^{f} \phi_{q}{ }^{0}\left|V_{p, q}^{\prime}\right| \Phi_{p}{ }^{0} \phi_{q}{ }^{f}\right\rangle-\left\langle\phi_{p}{ }^{f} \phi_{q}{ }^{0}\left|V_{p, q}\right| \phi_{p}{ }^{0} \phi_{q}{ }^{j}\right\rangle, \\
& \Delta_{p, n \alpha}{ }^{f}=\left\langle\bar{\phi}_{p}^{f} \phi_{n \alpha}{ }^{0}\left|V_{p, n \alpha}^{\prime}\right| \bar{\phi}_{p}{ }^{0} \phi_{n \alpha}{ }^{f}\right\rangle \\
& \text { and (see below) } \\
& -\left\langle\phi_{p}^{f} \phi_{n \alpha}{ }^{0}\left|V_{p, n \alpha}\right| \phi_{p}^{0} \phi_{n \alpha}{ }^{f}\right\rangle, \\
& \Delta_{q, q^{\prime}}{ }^{f}=\Delta_{q, n \alpha}{ }^{f}=\Delta_{n \alpha, n \alpha^{\prime}}{ }^{f}=0,
\end{aligned}
$$

where $\epsilon_{p}{ }^{f}=\left\langle\bar{\phi}_{p}{ }^{f}\left|H_{p}{ }^{\prime}\right| \bar{\phi}_{p}{ }^{f}\right\rangle-\left\langle\bar{\phi}_{p}{ }^{0}\left|H_{p}{ }^{\prime}\right| \bar{\phi}_{p}{ }^{0}\right\rangle$ is the gas phase transition energy of the guest at the $p$ th site. At this point we have grouped molecules in the crystal into guests (primary traps, denoted by $p$ ); perturbed hosts (secondary traps, denoted by $q$ ); and unperturbed hosts (denoted by $n \alpha$ ). The assumption that $\Delta_{n \alpha}{ }^{f} \approx 0$ is consistent with our model in which unperturbed hosts are very far away from primary traps, with negligible interactions with the traps. Equations (7) conveniently define primary traps, secondary traps and hosts. Primary traps are those with $\Delta_{p} f \neq 0$, $\Delta_{p, r} \neq 0 ;$ secondary traps are those with $\Delta_{q}^{f} \neq 0$, $\Delta_{q, r \neq p}{ }^{f}=0$; and hosts are those with $\Delta_{\mathrm{n} \alpha}{ }^{f}=\Delta_{\mathrm{n} \alpha, r \neq p} f=0$. $r$ is, of course, any molecule in the crystal.

It can be seen that the $D$ term plays an important role in the mixed crystal theory. On the part of the guest, the $D$ term shifts the gas phase trap depth, i.e., $\epsilon_{p} f-\epsilon_{h}^{f}$ to its corresponding mixed crystal value $\Delta_{p}{ }^{f}$. And, on the part of the host, the $D$ term is responsible for the creation of secondary traps with varying trap depth $\Delta_{q} f$. Equations (7) are slightly more general than the equations derived by Dubovskii and Konobeev, ${ }^{12}$ who ignored the secondary traps. It is also more general than those derived by Craig and Philpott, 11 who were primarily interested in the single-impurity problem.

The form of perturbation matrix can best be illustrated diagrammatically as shown in Fig. 1. It should be pointed out here that higher order perturbations, such as the perturbation on the interactions between secondary traps, have been completely ignored. This is evident both from Eqs. (7) and from Fig. 1. Although, in principle, the spheres of perturbations are infinitely extended, in reality, they can be truncated at certain distances. When such a truncation is made, the spheres of perturbations either overlap or remain separated, as indicated in Fig. 1.

It is now a simple matter to diagonalize the mixed crystal Hamiltonian, using the Koster and Slater ${ }^{13}$ scheme. Notice first that the mixed crystal wavefunctions $\Phi^{f}$ can be expressed in terms of the localized basis set as a column matrix:

$$
\Phi^{f}=\left(\begin{array}{c}
U^{f}\left(\mathbf{R}_{\mathrm{n} \alpha}\right) \\
U^{f}\left(\mathbf{R}_{\mathrm{m} \beta}\right) \\
\vdots
\end{array}\right),
$$


where $U^{\prime}\left(\mathbf{R}_{\mathrm{n} \alpha}\right)$ is the amplitude of the distorted exciton wave at site $\mathbf{R}_{\mathbf{n} \alpha}$, which is to be determined from the following matrix equation of the Schrodinger type:

$$
(E-\mathfrak{C}) \Phi^{f}=0
$$

Substituting Eq. (6) into Eq. (9) and rearranging, we have

or

$$
(E-H) \Phi^{f}=\Delta^{f} \Phi^{f}
$$

$$
\Phi^{f}=(E-H)^{-1} \Delta^{f} \Phi^{f}
$$

It follows immediately that the expression

$$
\operatorname{det}\left|1-(E-H)^{-1} \Delta^{f}\right|=0
$$

is the general equation for the mixed crystal levels outside the host band. Notice that $(E-H)^{-1}$, which is actually the pure crystal Green's function $G$, can be obtained through the use of the canonical transformation between the localized basis set and the delocalized basis set. Thus, we have ${ }^{14}$

$$
\begin{aligned}
& G_{\mathrm{n} \alpha, \mathrm{m} \beta} \equiv\left\langle\psi^{f}\left(\mathbf{R}_{\mathrm{n} \alpha}\right)\left|(E-H)^{-1}\right| \psi^{f}\left(\mathbf{R}_{\mathrm{m} \beta}\right)\right\rangle \\
&= N^{-1} \sum_{\mu} \sum_{k} B_{\mu \alpha} f(\mathbf{k}) \exp \left[i \mathbf{k} \cdot\left(\mathbf{R}_{\mathrm{n} \alpha}-\mathbf{R}_{\mathrm{m} \beta}\right)\right] \\
& \quad \times B_{\mu \beta^{f *}}(\mathbf{k})\left\langle\psi_{\mu}^{f}(\mathbf{k})\left|(E-H)^{-1}\right| \psi_{\mu}^{f}(\mathbf{k})\right\rangle \\
&= N^{-1} \sum_{\mu} \sum_{k}\left\{B _ { \mu \alpha } ^ { f } ( \mathbf { k } ) B _ { \mu \beta ^ { f * } } ( \mathbf { k } ) \operatorname { e x p } \left[i \mathbf { k } \cdot \left(\mathbf{R}_{\mathrm{n} \alpha}\right.\right.\right. \\
&\left.\left.\left.\quad-\mathbf{R}_{\mathrm{m} \beta}\right)\right] /\left[E-E_{\mu} f(\mathbf{k})\right]\right\},
\end{aligned}
$$

where $E_{\mu} f(\mathbf{k})$ is, of course, the eigenenergy of the pure crystal.

As we can see from Eqs. (7), the diagonal and offdiagonal elements of the perturbation matrix are due to perturbations on the static and dynamic interactions, respectively. Since they are derived from the same perturbation operator, it is safe to say that one always accompanies the other. In other words, if a host molecule sees a different static field after the introduction of an impurity, within our model, it means immediately that the dynamic coupling between the particular host and the particular impurity would also be different from that between two hosts. However, this does not preclude the possiblity that one of them might predominate. One can certainly envisage a situation where a secondary trap would interact similarly with an impurity and with a host. Conversely, one could also envisage a situation where an impurity causes no significant change in the static field while its dynamic coupling with the host is very different from the hosthost coupling. This latter case is of particular interest here because the secular equation, Eq. (11), can then be further reduced to one of smaller dimension.

Without any loss of generality, we first assume a perturbation of the form shown in Fig. 2. Included in the shaded area are all the primary and secondary traps. A secondary trap is being treated here as a special case of a primary trap. With such a perturbation, we

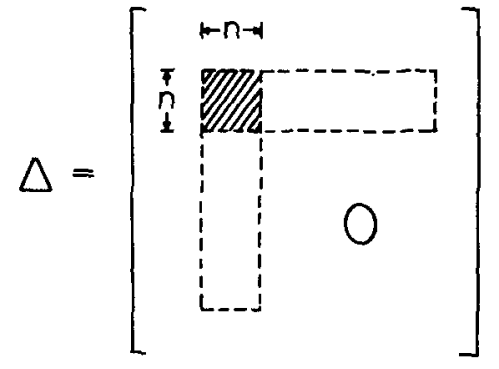

FIG. 2. A special form of perturbation matrix treated in the text. The off-diagonal perturbation is assumed to be infinitely extended whereas the diagonal perturbation has a limited range (within the $n \times n$ block). The shaded area includes all the primary and secondary traps.

can write

$$
\Delta^{f}=\left(\begin{array}{cc}
\Delta^{t t} & \Delta^{t s} \\
\Delta^{s t} & \Delta^{s s}
\end{array}\right),
$$

where $\Delta^{s s}$ is a null matrix (the index $f$ is supressed in the submatrices), $t$ refers to traps (primary and secondary) and $s$ to host. Similarly,

$$
G^{f}=\left(\begin{array}{ll}
G^{t t} & G^{t s} \\
G^{\mathrm{st}} & G^{s 8}
\end{array}\right),
$$

where $\Delta^{t t}$ and $G^{t t}$ are $n \times n$ matrices and $n$ is, of course, the total number of traps (primary and secondary). Now, for the trap amplitudes, $U^{f}\left(\mathbf{R}_{t}\right), \mathrm{Eq} .(10)$ can be displayed in the following way:

$$
\begin{aligned}
U^{f}\left(\mathbf{R}_{t}\right)=\sum_{t^{\prime}=1}^{n} \sum_{t^{\prime \prime}=1}^{n} G^{t t}{ }_{t t^{\prime}} \Delta^{t t_{t^{\prime \prime}}{ }_{t}} U^{f}\left(\mathbf{R}_{t^{\prime}}\right) \\
+\sum_{t^{\prime}=1}^{n} J_{t t^{\prime}} U^{f}\left(\mathbf{R}_{t^{\prime}}\right)+\sum_{t^{\prime}=1}^{n} G^{t t} t_{t^{\prime}} D_{t^{\prime}}
\end{aligned}
$$

where

$$
J_{t t^{\prime}} \equiv \sum_{s>n} G_{t s}^{t s} \Delta^{s t_{8 t^{\prime}}}
$$

and

$$
D_{i} \equiv \sum_{s>n} \Delta^{t_{s}}{ }_{t s} U^{f}\left(\mathbf{R}_{8}\right)
$$

$D_{t}$ can be further eliminated, if we make use of the expressions involving the host amplitudes. The host amplitudes are

$$
\begin{aligned}
& U^{f}\left(\mathbf{R}_{s}\right)=\sum_{t^{\prime}=1}^{n} \sum_{t^{\prime \prime}=1}^{n} G_{s t^{\prime \prime}}^{s t} \Delta^{t t_{i^{\prime \prime} t^{\prime}}} U^{f}\left(\mathbf{R}_{t^{\prime}}\right) \\
& +\sum_{t^{\prime}=1}^{n} \sum_{s^{\prime}>n} G_{s s^{\prime}}^{s s} \Delta_{s^{\prime} t^{\prime}}^{s t} U^{f}\left(\mathbf{R}_{t^{\prime}}\right)+\sum_{t^{\prime}=1}^{n} G^{s t}{ }_{s i} D_{t^{\prime}} .
\end{aligned}
$$

If we multiply both sides of Eq. (15) by $\Delta^{t s}{ }_{t s}$ and sum over all $s$, we obtain an expression involving only $D_{t}$ 
and $U^{f}\left(\mathbf{R}_{t}\right)$ :

$$
\begin{aligned}
D_{t}=\sum_{t^{\prime}=1}^{n} \sum_{t^{\prime \prime}=1}^{n} J^{\dagger}{ }_{t t^{\prime \prime}} \Delta^{t t}{ }_{t^{\prime \prime} t^{\prime}} U^{f}\left(\mathbf{R}_{t^{\prime}}\right) & \\
& +\sum_{t^{\prime}=1}^{n} J^{2}{ }_{t t^{\prime}} U^{f}\left(\mathbf{R}_{t^{\prime}}\right)+\sum_{t^{\prime}=1}^{n} J^{\dagger}{ }_{t t^{\prime}} D_{t^{\prime}},
\end{aligned}
$$

where

$$
J^{\dagger}{ }_{t t^{\prime}} \equiv \sum_{s>n^{\prime}} \Delta_{t s}^{t s} G_{8}^{s t} t_{s t^{\prime}}
$$

notice that

and

$$
J^{\dagger} t^{\prime}(z)=J_{t^{\prime} t^{*}}\left(z^{*}\right)
$$

$$
J^{2} t^{\prime} \equiv \sum_{s>n} \sum_{s^{\prime}>n} \Delta_{t s}^{t t_{s}} G_{s^{s}}^{s s_{s}} \Delta_{s^{\prime} t^{\prime}}
$$

Equations (14) and (16) can be written in a compact matrix form. The secular equation is now simply

$$
\operatorname{det}\left|1-\left(\begin{array}{cc}
G^{t t} \Delta^{t t}+J & G^{t t} \\
J^{\dagger} \Delta^{t t}+J^{2} & J^{\dagger}
\end{array}\right)\right|=0
$$

with a dimension equal to $2 n$. Notice that the matrix in Eq. (17) is non-Hermitian because the transformation from $U^{f}\left(R_{t}\right), U^{f}\left(R_{s}\right)$ to $U^{f}\left(R_{i}\right), D_{t}$ is not unitary.

In the case of only one primary trap and several secondary traps, it is easy to see that Eq. (17) can be reduced to the equations derived by Craig and Philpott, ${ }^{110}$ by noting that the $J$ matrix in Eq. (14b) has the following simple form (assuming that molecule 1 is a primary trap and molecules 2 to $n$ are secondary traps):

$$
J=\left(\begin{array}{cc}
J_{11} & \\
J_{21} & \\
& \\
J_{31} & 0 \\
\vdots & \\
J_{n 1} &
\end{array}\right) .
$$

To compare with Dubovskii and Konobeev's results, ${ }^{12}$ one should also note that Eqs. (14b) and (16c) can be rewritten as

$$
\begin{aligned}
J_{t t^{\prime}}=N^{-1} \sum_{\mu} \sum_{k} & \left\{B_{\mu t}(\mathbf{k}) A_{\mu t^{\prime}} *(\mathbf{k})\right. \\
& \left.\times \exp \left[i \mathbf{k} \cdot \mathbf{R}_{t}\right] /\left[E-E_{\mu}{ }^{f}(\mathbf{k})\right]\right\}
\end{aligned}
$$$$
\text { and }
$$

$J^{2}{ }^{\prime} t^{\prime}=N^{-1} \sum_{\mu} \sum_{k}\left\{A_{\mu t}(\mathbf{k}) A_{\mu t^{\prime}} *(\mathbf{k}) /\left[E-E_{\mu^{\prime}}{ }^{\prime}(\mathbf{k})\right]\right\}$,

where

$$
A_{\mu t^{\prime}} *(\mathbf{k})=\sum_{8} B_{\mu t} *(\mathbf{k}) \exp \left(-i \mathbf{k} \cdot \mathbf{R}_{s}\right) \Delta^{s t_{s}} \cdot
$$

In summary, the general mixed crystal problem is solved by first finding the eigenvalues from Eq. (17) and then the corresponding eigenvectors (in the form of linear combinations of $U^{f}\left(\mathbf{R}_{\ell}\right)$ and $\left.D_{t}\right)$. Subsequently, Eq. (15) can be used to calculate the $U^{f}\left(\mathbf{R}_{s}\right)$. The optical intensity of a particular cluster state $E^{\prime}$ is then simply

$$
I_{E^{\prime}} \propto\left|\sum_{t} U_{E^{\prime}} f\left(\mathbf{R}_{t}\right) \mathbf{d}_{t}+\sum_{s} U_{E^{\prime}}^{\prime}\left(\mathbf{R}_{s}\right) \mathbf{d}_{s}\right|^{2},
$$

where $d_{t}$ and $d_{s}$ are the transition moments of the trap and the host, respectively. $U_{E^{\prime}}{ }^{f}\left(\mathbf{R}_{t}\right)$ and $U_{E^{\prime}} f\left(\mathbf{R}_{\boldsymbol{s}}\right)$ are, of course, subject to the normalization condition, i.e.,

$$
\sum_{t}\left|U_{E^{\prime}} f\left(\mathbf{R}_{t}\right)\right|^{2}+\sum_{s}\left|U_{E^{\prime}} f\left(\mathbf{R}_{s}\right)\right|^{2}=1 .
$$

\section{A GROUP THEORETICAL DISCUSSION OF THE IMPURITY PROBLEM}

In the preceding section, we have intentionally adopted a molecular approach to the mixed crystal problem, rather than the more rigorous site approach. This was done mainly because of the simplicity involved in the former approach. The deviation from the pure crystal symmetries is attributed to a change in the $D$ term, a change in the off-diagonal elements, etc. Generally, these elements are very difficult to evaluate from $a b$ initio calculations. This is why most work on the impurity problem ignores such effects and, in an attempt to reduce the number of parameters, concentrates only on $\epsilon_{p}{ }^{j}-\epsilon_{h}{ }^{j}$, i.e., the gas-phase trap depth. This is also why most experimental work involves isotopic mixed crystals: These systems are the only systems for which such an approximation can be more-or-less justified. However, as is true in many other problems, caution must be exercised to see if any particular approach is consistent with the symmetry of the problem. Group theoretical discussions are thus important in this respect. Furthermore, in the absence of reliable $a b$ initio values for the aforementioned perturbations, symmetry arguments can at least be used to relate various elements of the perturbation matrix to one another.

In this section, we shall examine more closely the symmetry aspect of the impurity problem. In order of increasing complexity, we shall examine the singleimpurity problem, the "resonance pair" problem, the multicomponent cluster problem etc. We shall also illustrate how the formulation presented before can be related to the group theoretical arguments.

Mixed molecular crystals have some peculiar symmetry properties, especially when the host crystal has more than one molecule per primitive unit cell. Let us consider a simple single-impurity system such as naphthalene in durene, shown in Fig. 3. Notice that the following discussion can be applied to isotopic mixed crystals of naphthalene as well. The fact that the latter systems are simpler does not arise from pure symmetry arguments. As we can see from Fig. 3, the only symmetry element left in the mixed crystal is the inversion center at the guest site. In other words, $\Delta_{\mathrm{I}}{ }^{f}=\Delta_{\mathrm{I}^{\prime}}$, 
$\Delta_{\mathrm{II}}^{f}=\Delta_{\mathrm{II}}{ }^{f}$, but $\Delta_{\mathrm{I}^{f}} \neq \Delta_{\mathrm{II}}{ }^{f}$. This is built into the formulation in the preceding section, because $V_{0 \mathrm{II}} \neq V_{0 \mathrm{II}}{ }^{\prime}$, i.e.,

$$
\begin{aligned}
& \Delta_{\mathrm{I}}{ }^{f}=\left\langle\phi_{\mathrm{I}}{ }^{f} \bar{\phi}_{0}{ }^{0}\left|V^{\prime}{ }_{0 \mathrm{I}}\right| \phi_{\mathrm{I}}{ }^{f} \bar{\phi}_{0}{ }^{0}\right\rangle-\left\langle\phi_{\mathrm{I}}{ }^{f} \phi_{0}{ }^{0}\left|V_{0 \mathrm{I}}\right| \phi_{\mathrm{I}}{ }^{f} \phi_{0}{ }^{0}\right\rangle \\
& -\left\langle\phi_{\mathrm{I}}{ }^{0} \bar{\phi}_{0}{ }^{0}\left|V^{\prime}{ }_{0 \mathrm{I}}\right| \phi_{\mathrm{I}}{ }^{0} \bar{\phi}_{0}{ }^{0}\right\rangle+\left\langle\phi_{\mathrm{I}}{ }^{0} \phi_{0}{ }^{0}\left|V_{0 \mathrm{I}}\right| \phi_{\mathrm{I}}{ }^{0} \phi_{0}{ }^{0}\right\rangle \\
& \not 夫\left\langle\phi_{\text {II I }}{ }^{f} \bar{\phi}_{0}{ }^{0}\left|V^{\prime}{ }_{0 I I}\right| \phi_{I I}{ }^{f} \bar{\phi}_{0}{ }^{0}\right\rangle-\left\langle\phi_{I I}{ }^{f} \phi_{0}{ }^{0}\left|V_{0 I I}\right| \phi_{I I}{ }^{f} \phi_{0}{ }^{0}\right\rangle \\
& -\left\langle\phi_{\mathrm{II}}{ }^{0} \bar{\phi}_{0}{ }^{0}\left|V^{\prime}{ }_{0 \mathrm{II}}\right| \phi_{\mathrm{II}}{ }^{0} \bar{\phi}_{0}{ }^{0}\right\rangle+\left\langle\phi_{\mathrm{II}}{ }^{0} \phi_{0}{ }^{0}\left|V_{0 \mathrm{II}}\right| \phi_{\mathrm{II}}{ }^{0} \phi_{0}{ }^{0}\right\rangle \\
& =\Delta_{\mathrm{II}}{ }^{f} \text {. }
\end{aligned}
$$

When the approximation of an isotopic independent site shift can be justified, such as in the case of isotopic mixed crystals of naphthalene, ${ }^{15}$ then $\Delta_{\mathrm{I}}{ }^{f}=\Delta_{\mathrm{I}}{ }^{f}=\Delta_{\mathrm{II}}{ }^{f}=$ $\Delta_{\mathrm{II}}{ }^{f}=0$. We then retrieve the simple system of primary trap only, not from group theory but from physical approximations. It has been stated that for isotopic mixed crystals of benzene ${ }^{16}$ site shifts are isotope dependent and we have to assume that probably $V^{\prime}$ does not equal $V$. It is our opinion that secondary traps would be important in such systems, although no experimental data have as yet been obtained. However, as we pointed out in the introduction, secondary traps do play an important role in the isotopic mixed crystals of pyrazine. Recent work by Fischer $^{17}$ on $s$-triazine also supports this view. Presumably, the relatively strong interactions due to hydrogen bonding might be the source of such a perturbation.

Let us consider the two-impurity problem. We are particularly interested in the so-called "interchange pairs". 8,50 As pointed out by Hong and Kopelman, translational pairs are genuinely resonance pairs if the host lattice and the guest molecules both have inversion centers. This is not true for interchange pairs. Referring to Fig. 4, we see that although $\epsilon_{A} f=\epsilon_{A^{\prime}}$, the $D$ term lifts the degeneracy. In fact, according to Eqs. (7), and a "little reflection",

$$
\begin{aligned}
& \Delta_{A} f-\Delta_{A}, f=\left\langle\Phi_{A^{\prime}}{ }^{\prime} \bar{\phi}_{A^{\prime}},\left|V^{\prime \prime}{ }_{A A^{\prime}}\right| \bar{\phi}_{A^{\prime}}{ }^{\prime} \bar{\phi}_{A^{\prime}}, 0\right\rangle
\end{aligned}
$$

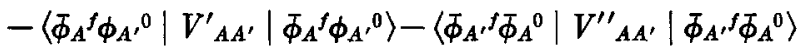

$$
\begin{aligned}
& +\left\langle\bar{\phi}_{A^{\prime}}{ }^{I} \phi_{A^{0}}\left|V^{\prime}{ }_{A A^{\prime}}\right| \bar{\phi}_{A}{ }^{f} \phi_{A^{0}}\right\rangle \neq 0 \text {. }
\end{aligned}
$$

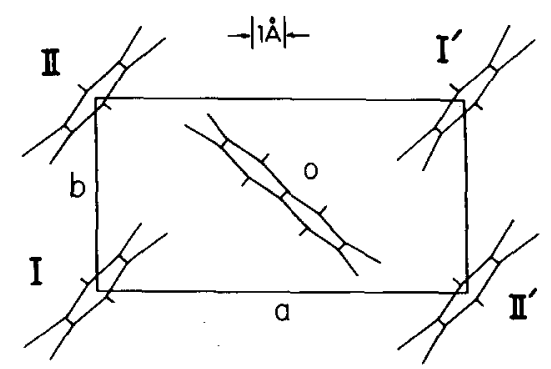

Fig. 3. A schematic drawing of naphthalene-in-durene mixed crystal. The guest is located at the origin $(O)$. Notice that I and II are related by symmetry to $\mathrm{I}^{\prime}$ and $\mathrm{II}^{\prime}$, respectively, by the inversion center at the guest site.

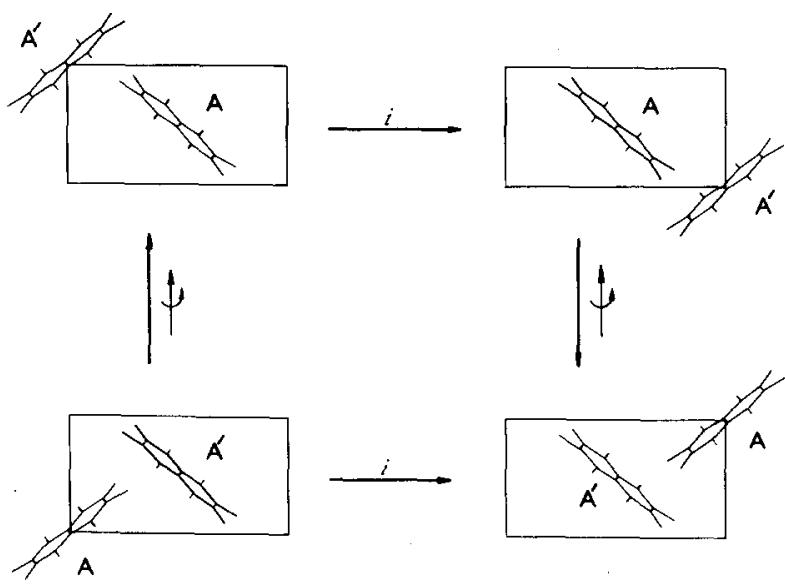

FIG. 4. A schematic drawing of a naphthalene interchange pair in a durene host lattice. Notice that the four configurations shown here are related by either screw-axis or inversion to each other and hence they are physically identical. However, $A$ and $A^{\prime}$ are nonsuperimposable, i.e., by symmetry, $\Delta_{\boldsymbol{A}} f \neq \Delta_{A^{\prime}} f$. See text.

Thus the simple molecular approach predicts the nonidentity of the $D$ term. Again, if $V^{\prime \prime}{ }_{A A^{\prime}}=V_{A^{\prime}}^{\prime}$ (not necessarily equal to $\left.V_{A A^{\prime}}\right)$ and $\bar{\phi}^{0} \approx \phi^{0}$, as is generally assumed for isotopic mixed crystals of naphthalene, we have "resonant" interchange pairs. It should also be pointed out here that for isotopic mixed crystals of naphthalene, if $\Delta_{A} f=\Delta_{A},{ }^{\prime}$, the excitation amplitude at $A$ and $A^{\prime}$ are always equal, although only in the restricted Frenkel limit ${ }^{18}$ does the distorted exciton contain a pseudoinversion. ${ }^{50}$ Furthermore, as long as $\Delta_{A}{ }^{f}=\Delta_{A^{\prime}}{ }^{f}$, theory predicts that interchange dimers yield uniquely polarized absorptions, be it restricted or general Frankel excitons. ${ }^{19}$

Also shown in Fig. 4 are some of the symmetry operations, which do not map $A$ onto $A^{\prime}$ but rather relate different possible arrangements of pairs. Since $A$ and $A^{\prime}$ are compositionally identical, Fig. 4 indicates that there is only one possible pair. In short, we have one distinct pair which is, strictly speaking, not in resonance.

The situation is different in a multicomponent cluster, which consists of, say, a naphthalene- $h_{8}$ molecule and a naphthalene- $d_{8}$ molecule in durene shown in Fig. 5. Two distinct pairs can be obtained. No symmetry operation would relate the arrangement in Fig. 5(a) to that in Fig. 5(b). It is apparent that $\Delta_{B} f$ in System 5(a) is not equal to $\Delta_{B}{ }^{\prime}$ in System 5(b) and the same is true for $\Delta_{C}{ }^{f}$ and $\Delta_{C}{ }^{f}$. If we are willing to make the asumption of isotopic invariance of such site effects, then $B, B^{\prime}$ and $C, C^{\prime}$ are not different from $A$ or $A^{\prime}$ in Fig. 4 , and we can see that, on the one hand, the transition energies for $B$ and $C$ in system $5(a)$ are separated by

$$
\epsilon_{B}^{f}-\epsilon_{C}^{f}+\left(\Delta_{A}^{f}-\Delta_{A^{\prime}} f\right),
$$

whereas, for System 5(b), they are separated by

$$
\epsilon_{B}^{f}-\epsilon_{C}^{f}-\left(\Delta_{A}^{f}-\Delta_{A^{\prime}}\right) \text {. }
$$




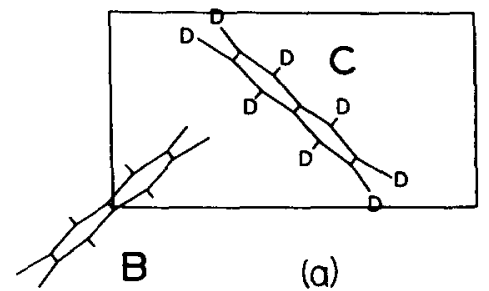

(a)

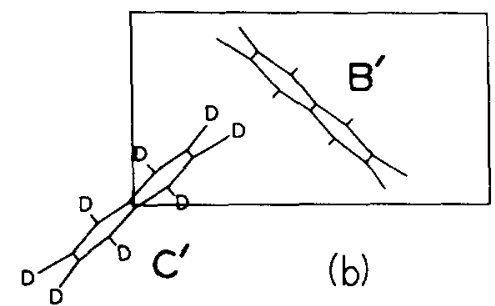

FIG. 5. A schematic drawing of a naphthalene-perdeutero-naphthalene mixed cluster in a durene host. (a) and (b) are distinct entities and hence two distinct interchange pairs can be formed. Notice also that $\Delta_{C} f \neq \Delta_{C^{\prime}} f$ and $\Delta_{B} f \neq \Delta_{B}$, . See text.
The study of multicomponent clusters might provide a means of detecting such a subtle site effect. Of course, in practice, one would probably like to eliminate the possible complications due to dynamic couplings between traps. In this respect, the study of triplet states appears to be more convenient than that of the singlet states.

We have discussed some symmetry properties of mixed crystals, using the nonsymmorphic monoclinic crystals of durene and naphthalene with two molecules per unit cell as examples. The purpose is to illustrate how the formulation in Sec. II can be used in conjunction with the symmetry arguments. More complicated crystals such as benzene, with four molecules per unit cell, can also be discussed within the same framework. Particular emphasis has been put on the importance of the $D$ term. The discussion also serves to demonstrate the fruitfullness of the molecular approach adopted here.

\section{APPLICATIONS TO THE ISOTOPIC MIXED CRYSTAL OF NAPHTHALENE: THE SUPEREXCHANGE EFFECT}

As we have mentioned earlier, for the general mixed crystal, the perturbations of the pure crystal Hamiltonian consist of two parts: First, the creation of secondary traps due to a change in the $D$ terms and, second, the difference between guest-host and hosthost excitation transfer interactions. For lack of reliable estimates of such perturbations, both theoretical and experimental, we limit our discussion in this section to the simple case of isotopic mixed crystals. Specifically, we discuss the mixed crystals of naphthalene for which experimental data have been obtained by Hanson: ${ }^{\text {s }}$

The perturbation matrix is especially simple for isotopic mixed crystals, namely,

$$
\Delta=\left(\begin{array}{cccc}
\Delta_{1} & 0 & 0 & \cdots \\
0 & \Delta_{2} & 0 & \cdots \\
0 & 0 & \Delta_{3} & \cdots \\
\cdot & \cdot & \cdot & \cdots \\
\cdot & \cdot & \cdot & \cdots \\
\cdot & \cdot & \cdot & \cdots
\end{array}\right)
$$

where $\Delta_{1}, \Delta_{2}, \Delta_{3}$ etc., are trap-depths of various guests.
The secular equation [Eq. (11)] now becomes

$$
\operatorname{det}\left(\begin{array}{cccc}
1-G_{11} \Delta_{1} & -G_{12} \Delta_{2} & -G_{13} \Delta_{3} & \cdots \\
-G_{21} \Delta_{1} & 1-G_{22} \Delta_{2} & -G_{23} \Delta_{3} & \cdots \\
-G_{31} \Delta_{1} & -G_{32} \Delta_{2} & 1-G_{33} \Delta_{3} & \cdots \\
\cdot & \cdot & \cdot & \cdots \\
\cdot & \cdot & \cdot & \cdots \\
\cdot & \cdot & \cdot & \cdots
\end{array} \mid=0 .\right.
$$

Notice that

$$
G_{11}=G_{22}=G_{33}=\cdots=\sum_{\mu} \sum_{k} N^{-1}\left[E-E_{\mu}(\mathbf{k})\right]^{-1} .
$$

Furthermore, $G_{n \alpha, m \beta}=G_{m \beta, n \alpha}$ [by definition, $G_{n \alpha, m \beta}(z)=$ $\left.G_{m \beta, n \alpha}{ }^{*}\left(z^{*}\right)\right]$ since the Green's functions for centrosymmetric crystals are real outside the band due to time reversal symmetry. ${ }^{20}$

In a mixed crystal of naphthalene- $h_{8}$ in naphthalene$d_{8}$, a second guest, ${ }^{13} \mathrm{C}^{12} \mathrm{C}_{9} \mathrm{H}_{8}$, is always present because of the natural abundance of ${ }^{13} \mathrm{C}$. Normally a third guest, $\mathrm{C}_{10} \mathrm{D}_{7} \mathrm{H}(\alpha$ or $\beta$ ), is also present as an isotopic impurity of the host. Mass spectral analysis indicates

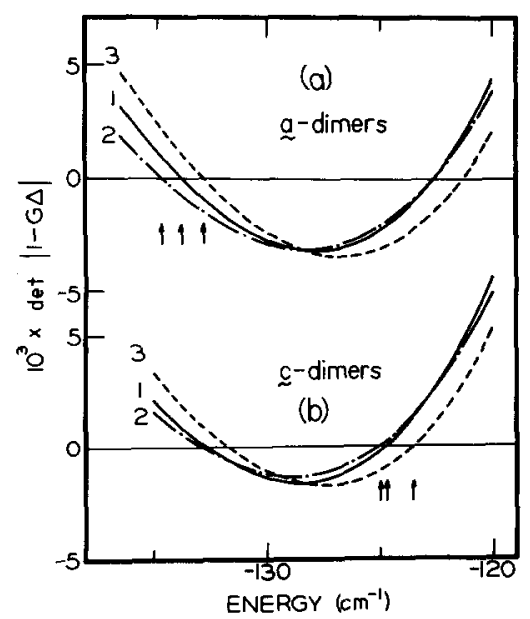

FIG. 6. The location of multicomponent cluster states by solving Eq. (24). Curves 1, 2, 3 are for a $\mathrm{C}_{10} \mathrm{H}_{8}-\mathrm{C}_{10} \mathrm{H}_{8}$ resonance pair, a $\mathrm{C}_{10} \mathrm{H}_{8}-\mathrm{C}_{10} \mathrm{H}_{8}-\mathrm{C}_{10} \mathrm{D}_{7} \mathrm{H}$ cluster and a $\mathrm{C}_{10} \mathrm{H}_{8}{ }^{-13} \mathrm{C}^{12} \mathrm{C}_{9} \mathrm{H}_{8}$ pair, respectively. The plus states are marked by arrows and the minus states are unmarked. Notice how the superexchange effect is perturbed differently by $D_{7}$ molecules for the a and $c$ dimers. See text. The minus state of a dimer is not perturbed by the presence of a $D_{7}$ molecule because of symmetry. 


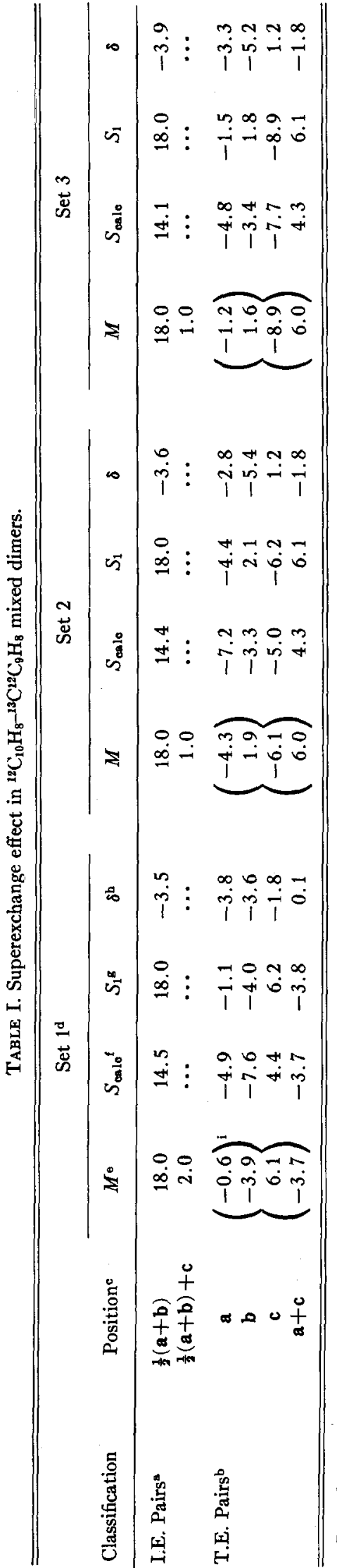

that a typical sample of naphthalene- $d_{8}$ (from Merck, Sharp and Dohme) contains from 16 to 20 mole percent of monohydronaphthalenes. As we show later, some fine structure reported by $\mathrm{Hanson}^{\text {5a }}$ can be assigned to the effect of this third guest.

In a previous paper by Hong and Kopelman, ${ }^{50}$ some elements of the Green's function for the ${ }^{1} B_{2 u}$ naphthalene exciton state were calculated within the restricted Frenkel formulation. Resonance pair states of naphthalene- $h_{8}$ in naphthalene- $d_{8}$ were analyzed and the dispersion relation was obtained. Here we report calculations of the cluster states involving the ${ }^{12} \mathrm{C}_{10} \mathrm{H}_{8}$ ${ }^{13} \mathrm{C}^{12} \mathrm{C}_{9} \mathrm{H}_{8}$ and $\mathrm{C}_{10} \mathrm{H}_{8}-\mathrm{C}_{10} \mathrm{D}_{7} \mathrm{H}$ mixed pairs and the $\mathrm{C}_{10} \mathrm{H}_{8}-\mathrm{C}_{10} \mathrm{H}_{8}-\mathrm{C}_{10} \mathrm{D}_{7} \mathrm{H}$ mixed trimer in a naphthalene- $d_{8}$ host lattice. The various elements of the Green's function were first evaluated from Eq. (12) through the use of the dispersion relation. These elements were then used to evaluate the determinant in Eq. (24). The cluster states were located as the zeros of the determinant. Some of these plottings are shown in Fig. 6 . All three sets of dispersion relation $\mathrm{s}^{50}$ were used and 16000 points in the first Brillouin zone were included. As before, we have assumed the ${ }^{1} B_{2 u}$ naphthalene band to be a restricted Frenkel exciton band. For ${ }^{13} \mathrm{C}^{12} \mathrm{C}_{9} \mathrm{H}_{8}$ in $\mathrm{C}_{10} \mathrm{D}_{8}$ a trap-depth of $-112.5 \mathrm{~cm}^{-1}$ was estimated from the known absorption spectrum ${ }^{\text {5a }}$ through the use of Koster and Slater's formula for single impurity states. We have not explicitly calculated the intensities for the clusters because their optical properties are similar to the corresponding unperturbed monomers or resonance pairs which have been discussed in a previous paper. ${ }^{\text {bo }}$

For $\mathrm{C}_{10} \mathrm{D}_{7} \mathrm{H}, \mathrm{McClure}^{21}$ has reported -11 and -19 $\mathrm{cm}^{-1}$ as trap-depths relative to $\mathrm{C}_{10} \mathrm{D}_{8}$ in a durene host lattice. We used only the $-19 \mathrm{~cm}^{-1}$ component (either $\alpha$ or $\beta$ ) here because it would perturb more strongly the naph thalene- $h_{8}$ molecules.

The $\mathrm{C}_{10} \mathrm{H}_{8}{ }^{13} \mathrm{C}^{12} \mathrm{C}_{9} \mathrm{H}_{8}$ dimers are near-resonance pairs. Within a first order approximation, the energy eigenstates can be easily obtained by solving the following secular equation ${ }^{22}$ :

$$
\begin{gathered}
\left|\begin{array}{cc}
-\frac{1}{2} \delta-E & W \\
W & \frac{1}{2} \delta-E
\end{array}\right|=0, \\
E= \pm\left(\frac{1}{4} \delta^{2}+W^{2}\right)^{1 / 2},
\end{gathered}
$$

where the energies are measured from the mean of the two individual monomer energies. Notice that for the translationally equivalent pairs the plus states have most of the intensity whereas for the interchange pair the plus states are nearly uniquely polarized along the b axis. ${ }^{23}$ Furthermore,

$$
\begin{array}{ll}
E_{+}=\left(\delta^{2} / 4+W^{2}\right)^{1 / 2} & \text { if } W>0, \\
E_{+}=-\left(\delta^{2} / 4+W^{2}\right)^{1 / 2} & \text { if } W<0 .
\end{array}
$$

When higher order terms were taken into account, we 


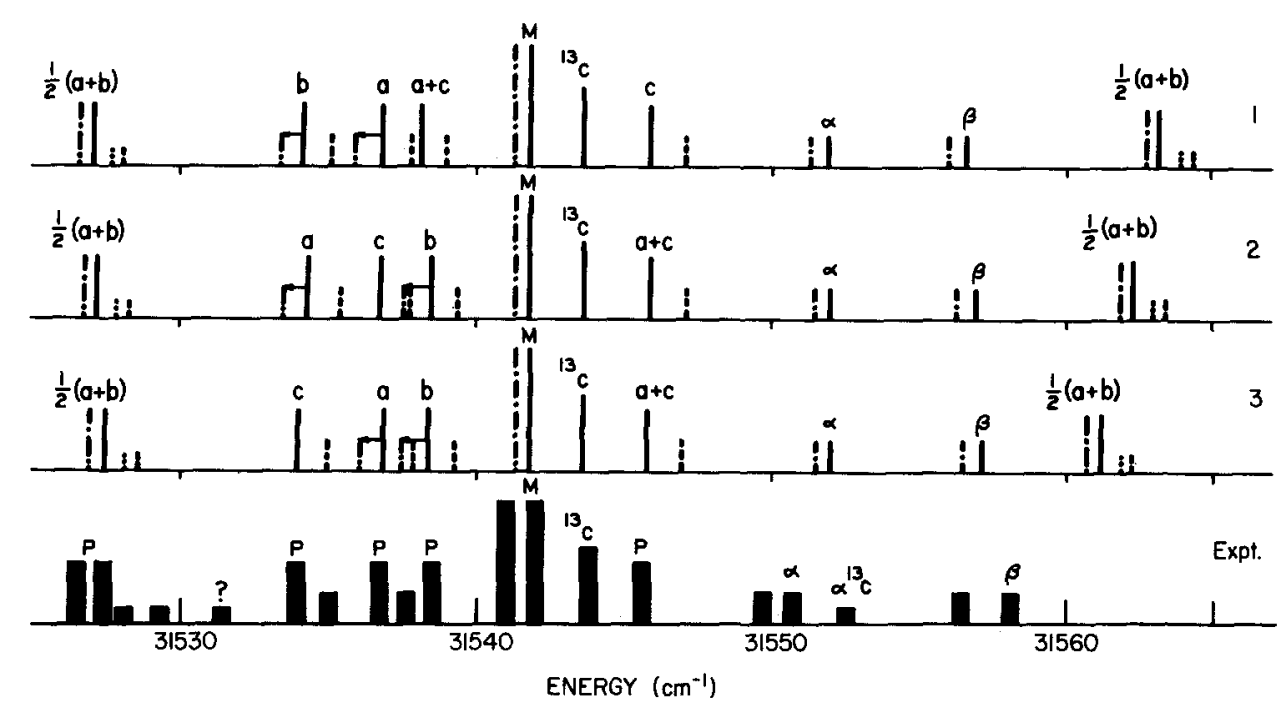

Frg. 7. A comparison of our calculations with the experimental data of Hanson [Ref. 5(a)]. The different symbols are: $M$, monomer; ${ }^{13} \mathrm{C},{ }^{18} \mathrm{C}^{12} \mathrm{C}_{9} \mathrm{H}_{8} ; P$, pair; $\alpha, \alpha \mathrm{C}_{10} \mathrm{H}_{7} \mathrm{D} ; \beta, \beta \mathrm{C}_{10} \mathrm{H}_{7} \mathrm{D} ; \mathrm{a}, \mathbf{b}, \mathbf{c}, \mathbf{a}+\mathbf{c}$, and $\frac{1}{2}(\mathrm{a}+\mathbf{b})$, translational pairs $\mathrm{a}, \mathrm{b}, \mathrm{c}$, (a+c), and interchange pair; ?, unassigned; $1,2,3$; sets 1, 2, 3 of pairwise interactions from Ref. $5(\mathrm{c})$. The mixed ${ }^{13} \mathrm{C}$ pair states are denoted by -- - and the mixed $D_{7}$ dimer and trimer states are denoted by - - . The one marked by $\cdots$ is a ${ }^{13} \mathrm{C}^{12} \mathrm{C}_{8} \mathrm{H}_{8}-\mathrm{C}_{10} \mathrm{H}_{8}-\mathrm{C}_{10} \mathrm{D}_{7} \mathrm{H}$ mixed cluster state. The heights represent schematically the concentrations. A line-width of $0.6 \mathrm{~cm}^{-1}$ is used here, which approximately equals the experimental width. The theoretical values obtained in this work and shown here agree with our previous calculations [Ref. 5 (c)] to within $0.2 \mathrm{~cm}^{-1}$. Note also that the high-energy $\frac{1}{2}(a+b)$ component was observed by Hanson as a continuous background absorption, the broadening being attributed by him to isotopic impurities. To emphasize the perturbation on the superexchange effect we have connected the $D_{7}$ perturbed dimer states with their parent dimer states by arrows. Only perturbed $\mathbf{a}$ and $\mathbf{b}$ translational dimer states are shown here because these dimers are perturbed most by the $D_{7}$ molecules.

observed the similar guest-host-guest couplings which we called the "superexchange effect". ${ }^{\circ}$ In Table I, we compared our results by solving Eq. (24) and those obtained from Eq. (25b). Notice that in each case, the superexchange contributions, defined as the differences between the rigorous Eq. (24) and the approximate Eq. (25a), are comparable with our previous results on resonance pairs. For $\mathbf{a}$ and $\mathbf{b}$ mixed dimers, such corrections are considerably larger than for $\mathrm{c}$ and $\mathrm{a}+\mathrm{c}$ dimers. Because the superexchange effects on the nearresonance pairs are quite similar to the superexchange effects on the resonance pairs, ${ }^{\text {bc }}$ we found that the $\mathrm{C}_{10} \mathrm{H}_{8}{ }^{-13} \mathrm{C}^{12} \mathrm{C}_{9} \mathrm{H}_{8}$ levels were separated from the corresponding $\mathrm{C}_{10} \mathrm{H}_{8}-\mathrm{C}_{10} \mathrm{H}_{8}$ levels by roughly $1 \mathrm{~cm}^{-1}$ (i.e., $\delta / 2$ ). Hanson ${ }^{\text {ba }}$ used this as his criterion for assigning the mixed pairs. However, it should be pointed out that when $\delta / 2$ is not small compared with the trapdepths of the two guests, this criterion would lead to erroneous results. As we show in Fig. 7, the rigorous treatment of the $\mathrm{C}_{10} \mathrm{H}_{8}{ }^{13} \mathrm{C}^{12} \mathrm{C}_{9} \mathrm{H}_{8}$ mixed pairs leads to satisfactory agreements with the observed absorptions. (However, vide infra.) As we pointed out earlier, the minus states of the translational pairs have very weak intensity, because of the near-resonance condition, this is why only the plus states are included in Table I.

It is convenient to consider the $\mathrm{C}_{10} \mathrm{H}_{8}-\mathrm{C}_{10} \mathrm{D}_{7} \mathrm{H}$ pair states as the $\mathrm{C}_{10} \mathrm{H}_{8}$ monomer states perturbed by $\mathrm{C}_{10} \mathrm{D}_{7} \mathrm{H}$. Similarly, the $\mathrm{C}_{10} \mathrm{H}_{8}-\mathrm{C}_{10} \mathrm{H}_{8}-\mathrm{C}_{10} \mathrm{D}_{7} \mathrm{H}$ trimer states can be regarded as perturbed $\mathrm{C}_{10} \mathrm{H}_{8}-\mathrm{C}_{10} \mathrm{H}_{8}$ pair states. When the perturbations are small, as in cases where $\mathrm{C}_{10} \mathrm{D}_{7} \mathrm{H}$ molecules are weakly coupled to the parent monomer or dimer states, the respective monomer or dimer states would simply be broadened. This is true when $\mathrm{C}_{10} \mathrm{D}_{7} \mathrm{H}$ molecules are coupled to the monomers or dimers via any interactions other than the large $M_{12}$ [interchange $\frac{1}{2}(a+b)$ interaction]. We have investigated various mixed $\mathrm{C}_{10} \mathrm{H}_{8}-\mathrm{C}_{10} \mathrm{D}_{7} \mathrm{H}$ dimers and mixed $\mathrm{C}_{10} \mathrm{H}_{8}-\mathrm{C}_{10} \mathrm{H}_{8}-\mathrm{C}_{10} \mathrm{D}_{7} \mathrm{H}$ trimers. For the mixed pairs it is found that only when $\mathrm{C}_{10} \mathrm{D}_{7} \mathrm{H}$ is situated at $\frac{1}{2}(a+b)$ does the mixed dimer state differ from the monomer state by $0.5 \mathrm{~cm}^{-1}$. Among the trimer states only the $\mathbf{a}$ and $\mathbf{b}$ dimers are perturbed by as much as $1 \mathrm{~cm}^{-1}$ when the $\mathrm{C}_{10} \mathrm{D}_{7} \mathrm{H}$ molecules are coupled via the large $M_{12}$ interaction. For the c and $\mathrm{a}+\mathrm{c}$ dimers, moderate perturbations were observed for the following configurations: $r_{1}=0, r_{2}=c, r_{3}=\frac{1}{2}(b-a)$; and $r_{1}=0$, $r_{2}=\mathbf{a}+\mathbf{c}, r_{3}=\frac{1}{2}(\mathbf{a}+\mathbf{b})$. Even in these configurations the perturbation shifts the dimer levels by less than 0.5 $\mathrm{cm}^{-1}$.

In Fig. 6, calculations on the $\mathrm{C}_{10} \mathrm{H}_{8}-\mathrm{C}_{10} \mathrm{H}_{8}$ resonance pair states, the $\mathrm{C}_{10} \mathrm{H}_{8}{ }^{13} \mathrm{C}^{12} \mathrm{C}_{9} \mathrm{H}_{8}$ mixed pair states and $\mathrm{C}_{10} \mathrm{H}_{8}-\mathrm{C}_{10} \mathrm{H}_{8}-\mathrm{C}_{10} \mathrm{D}_{7} \mathrm{H}$ mixed trimer states are shown for the $\mathbf{a}$ and $\mathbf{c}$ dimers. The locations of the plus states were indicated by arrows. Notice that the minus state of the a dimers is not perturbed by the presence of $\mathrm{a} \mathrm{C}_{10} \mathrm{D}_{7} \mathrm{H}$ molecule because of symmetry. As mentioned before, the $\mathrm{C}_{10} \mathrm{H}_{8}-{ }^{13} \mathrm{C}^{12} \mathrm{C}_{9} \mathrm{H}_{8}$ mixed pairs states are always located $1 \mathrm{~cm}^{-1}$ higher from the resonance pair states. Therefore, 
TABLE II. The host Davydov components and the host band centers as predicted by the three sets of pairwise interactions. ${ }^{\mathrm{a}}$

\begin{tabular}{|c|c|c|c|c|c|}
\hline & $E_{m}^{\mathrm{b}}$ & $E_{a c}$ & $E_{b}$ & $E_{\text {mean }}{ }^{\mathrm{c}}$ & $E_{\text {B.c. }}{ }^{d}$ \\
\hline Set 1 & 31541.8 & 31588.4 & 31748.4 & 31668.4 & 31670.6 \\
\hline Set 2 & 31541.8 & $31589.3^{t}$ & $31741.3^{t}$ & 31665.3 & 31670.3 \\
\hline Set 3 & 31541.8 & $31588.9 \mathrm{~g}$ & $31740.9 \mathrm{~g}$ & 31664.9 & 31669.9 \\
\hline Expte & 31541.8 & 31587.7 & 31751.0 & 31669.4 & $31671.4^{\mathrm{h}}$ \\
\hline
\end{tabular}

When we place the monomer energies all at $31541.8 \mathrm{~cm}^{-1}$; all energies in units $\mathrm{cm}^{-1}$.

b The monomer energies.

- $E_{\text {mean }}=\frac{1}{3}\left(E_{a c}+E_{b}\right)$.

${ }^{d} E_{\text {B.C. }}=$ band center.

- See, e.g., Ref. 15

If the $\frac{1}{2}(\mathrm{a}+\mathrm{b})+\mathrm{c}$ interaction is taken to be $2 \mathrm{~cm}^{-1}$ instead of $1 \mathrm{~cm}^{-1}, E_{a c}=31585.3$ and $E_{b}=31745.3$. This slight change would not affect other calculations.

If the same change were made as in (f) $E_{a c}=31584.9$ and $E_{b}=31744.9$.

b Calculated from $E_{\text {mean }}$ by assuming a translational shift of $-2 \mathrm{~cm}^{-1}$, according to Ref. 25 .

these mixed pair states are not useful in assigning the translational pair states to either $\operatorname{set}^{\text {bo }} 1,2$, or 3. However, for the $\mathrm{C}_{10} \mathrm{D}_{7} \mathrm{H}$ perturbed states (the mixed trimer states), the a perturbed dimer is separated from its parent state by almost twice the distance we find between the $c$ perturbed dimer state and its parent state. In summary, it is concluded that trimer states would not be observed except those corresponding to the $\mathbf{a}$ and $\mathbf{b}$ dimers perturbed by $\mathrm{C}_{10} \mathrm{D}_{7} \mathrm{H}$ via the $M_{12}$ term. These results also indicate that the superexchange effect could be utilized in separating the $a$ or $b$ dimers from the $\mathbf{c}$ or $\mathbf{a}+\mathbf{c}$ dimers. In other words, if the mixed trimer states were studied carefully, the parent dimer states which are largely perturbed due to the presence of $\mathrm{C}_{10} \mathrm{D}_{7} \mathrm{H}$ must be assigned as $\mathbf{a}$ or $\mathbf{b}$ dimers. In our previous study, ${ }^{\text {b }}$ we have utilized the superexchange effect to exclude three out of six possible assignments. Further studies would enable us to distinguish among the remaining three.

In reference to the experimental data of Hanson, ${ }^{5 \mathrm{a}}$ we encountered some ambiguities in assigning the fine structures around the translational pair lines. These difficulties arise because the $\mathrm{C}_{10} \mathrm{H}_{8}-\mathrm{C}_{10} \mathrm{H}_{8}-\mathrm{C}_{10} \mathrm{D}_{7} \mathrm{H}$ trimer systems really belong to the realm of heavilydoped mixed crystal problems (each $\mathrm{C}_{10} \mathrm{D}_{7} \mathrm{H}$ is present in about $8 \%-10 \%$ ). At this stage, it is difficult to assess the relative importance of $\mathrm{C}_{10} \mathrm{H}_{8}{ }^{-13} \mathrm{C}^{12} \mathrm{C}_{9} \mathrm{H}$ and $\mathrm{C}_{10} \mathrm{H}_{8}-$ $\mathrm{C}_{10} \mathrm{H}_{8}-\mathrm{C}_{10} \mathrm{D}_{7} \mathrm{H}$ clusters at the particular concentrations these studies were made, although a crude estimate would put the former contribution as the slightly more important of the two. ${ }^{24}$ Notice that if we associated the 31537.6 and $31535.0 \mathrm{~cm}^{-1}$ lines with $a$ and $b$ dimers perturbed by $\mathrm{C}_{10} \mathrm{D}_{7} \mathrm{H}$, set 3 would be the correct assignment. This assignment would ignore the $\mathrm{C}_{10} \mathrm{H}_{8}$ ${ }^{13} \mathrm{C}^{12} \mathrm{C}_{9} \mathrm{H}$ contributions and is consistent with the fact that no ${ }^{13} \mathrm{C}$ lines associated with the 31538.0 and $31545.6 \mathrm{~cm}^{-1}$ lines were reported. On the other hand, Hanson's assignments would give better agreement for the $31535.0 \mathrm{~cm}^{-1}$ line which is 1.1 above the 31533.9 $\mathrm{cm}^{-1}$ one. It is also conceivable that ${ }^{13} \mathrm{C}$ mixed pairs and the $D_{7}$-perturbed dimers overlap with each other and are unresolved. In this case, the two assignments would not conflict with each other. Hanson proposed to study the enriched ${ }^{13} \mathrm{C}$ samples. It is clear that the study of either enriched $\mathrm{C}_{10} \mathrm{D}_{7} \mathrm{H}$ samples or of the "stripped" $\mathrm{C}_{10} \mathrm{D}_{8}$ samples would also help to resolve these ambiguities.

Hanson also reported splittings in the $\mathrm{C}_{10} \mathrm{H}_{8}, \alpha$ $\mathrm{C}_{10} \mathrm{H}_{7} \mathrm{D}$ and $\beta-\mathrm{C}_{10} \mathrm{H}_{7} \mathrm{D}$ monomer lines at low concentrations of $\mathrm{C}_{10} \mathrm{H}_{8}$. It appears reasonable to assign them as due to mixed $\mathrm{C}_{10} \mathrm{H}_{8}-\mathrm{C}_{10} \mathrm{D}_{7} \mathrm{H}$ states [with $r_{1}=0$ and $\left.r_{2}=\frac{1}{2}(a+b)\right]^{25}$ The calculated splittings are of the order of $0.5 \mathrm{~cm}^{-1}$, which is close to the observed 1.0 $\mathrm{cm}^{-1}$ splittings. The $\alpha-\mathrm{C}_{10} \mathrm{H}_{7} \mathrm{D}$ and $\beta-\mathrm{C}_{10} \mathrm{H}_{7} \mathrm{D}$ monomer states are complicated by the possible orientational effects. ${ }^{26}$ This might contribute partly to the observed splittings. In addition to these, four lines were observed around the interchange pair states. We tentatively assign them to the combined ${ }^{13} \mathrm{C}$ and $\mathrm{C}_{10} \mathrm{D}_{7} \mathrm{H}$ perturbations. The interchange pair is found to be effected largely when $\mathrm{C}_{10} \mathrm{D}_{7} \mathrm{H}$ is located at $\mathbf{a}, \mathbf{b}$, or $(\mathbf{a}+\mathbf{b})$, with $r_{1}=0, r_{2}=\frac{1}{2}(a+b)$. In each case, the perturbed dimer state is split from the unperturbed state by $0.5 \mathrm{~cm}^{-1}$. Notice that the interchange ${ }^{13} \mathrm{C}$ line was observed at slightly higher concentration (1.4\%) because the ac polarized component is weaker in naphthalene. At this concentration, it is estimated that the $D_{7}$ perturbed interchange pair has higher concentration than the ${ }^{13} \mathrm{C}$ mixed dimer. ${ }^{27}$

We have summarized the comparisons between our calculated results and the experimental data in Fig. 7. The monomer lines from all three sets were placed at $31541.8 \mathrm{~cm}^{-1}$ as before. ${ }^{\text {tc }}$ When this is done, each set would yield slightly different Davydov components and band centers for the host band. To facilitate other schemes of comparison we have tabulated these quanti- 
ties in Table II. In principle, a satisfactory set should, of course, compare well with all the available data. However, considering uncertainties in the hot band experiment $^{28}$ and in the determination of trap depths, ${ }^{29}$ the agreement appears to be good.

We have again demonstrated the usefulness of the superexchange concept. The translational pairs can be assigned by (1) studying the resonance pairs at various trap depths, (2) studying the resonance pairs perturbed by a second guest and possibly (3) by studying the resonance pairs under uniaxial stress, electric field etc. While the last method appears to be quite involved, the first two are simple and workable. The underlying principle always involves the superexchange effect discussed here. Finally, we should point out here that we have relaxed the rigorous symmetry requirements discussed in the preceding section when we treated the isotopic mixed crystal problem. For example, strict symmetry requirements would predict that $\Delta_{1} \neq \Delta_{2}$ for $r_{1}=0$ and $r_{2}=\mathbf{a}$ when $r_{3}=\frac{1}{2}(\mathbf{a}+\mathbf{b})$. Thus, more than one trimer would have to be treated. The multicomponent cluster problems would then become unmanagably complicated.

\section{6 .}

* Supported by NSF Grant GP-18718 and NIH Grant NSO-

† Present address: Department of Chemistry, State University of New York, Stony Brook, N.Y. 11790.

1 (a) J. Frenkel, Phys. Rev. 37, 17, 1276 (1931); (b) A. S. Davydov, Theory of Molecular Excitons (McGraw-Hill, New York, 1962); (c) A. S. Davydov, Usp. Fiz. Nauk 82, 393 (1964) [Sov. Phys. Usp. 7, 145 (1964)]; (d) A. S. Davydov, Theory of Molecular Excitons (Plenum, New York, 1971).

${ }^{2}$ For example, if both guest $A$ and guest $B$ are present in small amounts, the concentration of clusters, consisting of $A$ plus $B$, would be $C_{A} C_{B}$, which is smaller than either $C_{A}$ or $C_{B}$ in such limit.

${ }^{3} \mathrm{~J}$. Hoshen has discussed this problem within the coherent potential approximation. See J. Hoshen, Ph.D. thesis, Tel-Aviv University, 1971.

4 Since the impurity states inside the host band (or virtual states) are generally broad, no such states have been identified experimentally in dilute mixed crystals [however, see J. C. Laufer and R. Kopelman, J. Chem. Phys. 57, 3202 (1972)]. Theoretical treatments, however, are quite abundant in the literature. See, for example, B. S. Sommer and J. Jortner, J. Chem. Phys. 50, 187, 822 (1969); Y. A. Izyumov, Advan. Phys. 14, 569 (1965) . A. Shibatani and Y. Toyozawa, J. Phys. Soc. Japan 25, 335 (1968). In order to detect virtual states at low impurity concentration, an impurity with much larger oscillator strength than the host is called for. In other words, chemically mixed crystals rather than isotopically mixed crystals are more amenable to such an investigation. Mixed crystals with large concentration of very shallow traps have been studied by $\mathbf{H}$. K. Hong and G. W. Robinson [J. Chem. Phys. 52, 825 (1970); 54, 1369 (1971)]; however, it seems no longer proper to refer to the guest states as virtual states.

5 (a) D. M. Hanson, J. Chem. Phys, 52, 3409 (1970). (b) H. K. Hong and R. Kopelman, Phys. Rev. Letters 25, 1030 (1970). (c) H. K. Hong and R. Kopelman, J. Chem. Phys. 55, 724 (1971). ${ }^{6}$ (a) D. S. McClure and E. F. Zalewski, Molecular Luminescence, edited by E. C. Lim (Benjamin, New York, 1969).

(b) H. K. Hong (unpublished).

${ }^{7}$ H. K. Hong and R. Kopelman, J. Chem. Phys. 55, 5380 (1971).

${ }^{8}$ R. Kopelman, J. Chem. Phys. 47, 2631, 3227 (1967).

$\checkmark$ We have adopted the free molecule approach here because of its simplicity. The merit of such an approach will become clear after Sec. III. In the more rigorous site approach the $\phi$ 's would be the site functions and could be different for the same molecular species at different environments.
${ }^{10}$ The crystal Hamiltonian takes up different forms as a consequence of the different choices of origins. One could compare Ref. 1(b) and Ref. 1(d) to see such changes. See also p. 42 of Ref. 1(d).

it D. Craig and M. R. Philpott, (a) Proc. Roy. Soc. (London) A290, 583 (1966); (b) A290, 602 (1966); (c) A293, 213 (1966). ${ }_{12} \mathrm{O}$. A. Dubovskii and Y. V. Konobeev, Fiz. Tverd. Tela 6, 2599 (1964) [Sov. Phys., Solid State 6, 2071 (1965)].

${ }_{13}$ G. F. Koster and J. C. Slater, Phys. Rev. 95, 1167 (1954); 96, 1208 (1954); G. F. Koster, Phys. Rev. 95, 1436 (1954).

${ }^{14}$ See, for example, B. S. Sommer, and J. Jortner, J. Chem. Phys. 50, 187 (1969).

${ }^{15}$ There is mounting evidence that this it true. See, for example, D. M. Hanson, R. Kopelman, and G. W. Robinson, J. Chem. Phys. 51, 212 (1969).

${ }^{16}$ S. D. Colson, J. Chem. Phys. 48, 3324 (1968).

${ }_{17}$ G. Fischer, J. Chem. Phys. 57, 2646 (1972).

${ }_{18}$ S. D. Colson, R. Kopelman, and G. W. Robinson, J. Chem. Phys. 47, 27, 5462 (1967); G. W. Robinson, Ann. Rev. Phys. Chem. 21, 429 (1970).

${ }^{19}$ For restricted Frenkel case, see Ref. 5(c); for general Frenkel case, see B. S. Sommer and J. Jortner, J. Chem. Phys. 51, 5559 (1969).

${ }^{20}$ For time-reversal symmetry, see L. P. Bouckaert, R. Smoluchowski, and E. Wigner, Phys. Rev. 50, 58 (1936). Notice that

$$
\begin{aligned}
G_{n \alpha, m \beta}= & N^{-1} \sum_{\mu} \sum_{k} \\
& \quad \times\left\{B_{\mu \alpha}(\mathbf{k}) B_{\mu \beta} *(\mathbf{k}) \exp \left[i \mathbf{k} \cdot\left(\mathbf{R}_{n \alpha}-\mathbf{R}_{m \beta}\right)\right] /\left[E-E_{\mu}(\mathbf{k})\right]\right\} \\
= & N^{-1} \sum_{\mu} \sum_{\bar{k}} \\
\times & \left(2 \operatorname{Re}\left\{B_{\mu \alpha}(\overline{\mathbf{k}}) B_{\mu \beta} *(\overline{\mathbf{k}}) \exp \left[i \overline{\mathbf{k}} \cdot\left(\mathbf{R}_{n \alpha}-\mathbf{R}_{m \beta}\right)\right]\right\} /\left[E-E_{\mu}(\overline{\mathbf{k}})\right]\right)
\end{aligned}
$$

where $R e$ is the real part and $\bar{k}$ only runs through one half of the Brillouin zone. When the crystal is centrosymmetric, the $B_{\mu \alpha}(\mathbf{k})$ 's are all real and hence

$$
\begin{aligned}
G_{n \alpha, m \beta} & =N^{-1} \sum_{\mu} \sum_{\bar{k}} \frac{2 B_{\mu \alpha}(\overline{\mathbf{k}}) B_{\mu \beta}(\mathbf{k}) \cos \overline{\mathbf{k}} \cdot\left(\mathbf{R}_{n \alpha}-\mathbf{R}_{m \beta}\right)}{E-E_{\mu}(\overline{\mathbf{k}})} \\
& =N^{-1} \sum_{\mu} \sum_{\bar{k}} \frac{2 B_{\mu \beta}(\overline{\mathbf{k}}) B_{\mu \alpha}(\mathbf{k}) \cos \overline{\mathbf{K}} \cdot\left(\mathbf{R}_{m \beta}-\mathbf{R}_{n \alpha}\right)}{E-E_{\mu}(\overline{\mathbf{k}})} \equiv G_{m \beta, n \alpha .}
\end{aligned}
$$

For naphthalene, within the restricted Frankel limit

$$
\left|B_{\mu \alpha}(\mathbf{k})\right|=\left|B_{\mu \beta}(\mathbf{k})\right|=1 / \sqrt{2}
$$

for all $\mathrm{k}$ and $\mu$.

${ }^{21}$ D. S. McClure, J. Chem. Phys., 24, 1 (1956).

${ }^{22}$ See for example, G. Herzberg, Molecular Spectra and Molecular Structure. II. Infrared and Raman Spectra of Polyatomic Molecules (Van Nostrand, Princeton, N.J., 1945), p. 216 on Fermi resonance.

${ }^{23} \mathrm{We}$ have used the $\mathrm{C}_{2}$ interchange convention here. For more discussion on the interchange convention, see Ref. 8 .

${ }^{24} \mathrm{~A}$ crude estimation of the concentrations of ${ }^{13} \mathrm{C}$ mixed pairs and $D_{7}$ perturbed dimers can be made, if we assume that the cluster states are essentially determined by the local configurations. If we take the $D_{7}$ concentration to be $10 \%$, we find that, with $0.65 \%$ of $\mathrm{C}_{10} \mathrm{H}_{8}$ the concentrations of ${ }^{13} \mathrm{C}$ mixed $a$ and $b$ pairs are $1.66 \times 10^{-5}$ whereas the corresponding $D_{7}$ perturbed dimers are $1.52 \times 10^{-5}$. These estimates are crude because the $D_{7}$ concentrations vary and also because we ignore the effect of nonnearest neighbors on the cluster states.

${ }_{25}$ Again, if we use the same crude estimation as in Ref. 24 we find that $29 \%$ of the monomers are perturbed by the presence of a simgle $D_{7}$ molecule whereas $66 \%$ of them are unperturbed, assuming we have $10 \% D_{7}$.

${ }^{26}$ See Ref, 8 and also E. R. Bernstein, S. D. Colson, R. Kopelman, and G. W. Robinson, J. Chem. Phys. 48, 4832 (1968).

${ }^{27}$ In terms of absolute concentrations, we find that, with $1.4 \% \mathrm{C}_{10} \mathrm{H}_{8}$, we have $2.78 \times 10^{-4}$ perturbed interchange dimers (including $r_{3}=\mathbf{a}, \mathbf{b}$ and $\mathbf{a}+\mathbf{b}$ ) which is $35 \%$ of the total interchange pairs, whereas the concentration of ${ }^{13} \mathrm{C}$ mixed pairs is only $1.72 \times 10^{-4}$.

${ }^{28}$ S. D. Colson, D. M. Hanson, R. Kopelman and G. W. Robinson, J. Chem. Phys. 48, 2215 (1968).

${ }^{29}$ For a rather complete discussion on the determination of trap depths, see Ref. 15., which also includes an exhaustive tabulation of experimental data by various workers. 